\title{
Hybride Gesellschaften im Verhältnis Schweiz-Deutschland
}

Grundsätzlich treten im Staatenverhältnis SchweizDeutschland keine hybriden Gesellschaften auf, da die Rechtsformen und die jeweiligen steuerlichen Einordnungsmethodiken weitgehend korrespondieren. Jedoch sind vorgeschaltete hybride Gesellschaften mit einer Betriebsstätte in der Schweiz möglich. Sie können für ausländische Investoren attraktiv sein und steuerliche Vorteile mit sich bringen. Der vorliegende Beitrag befasst sich mit der Einordnung und Besteuerung von hybriden Gesellschaften im Verhältnis Schweiz-Deutschland und insbesondere mit dem in der Literatur bisher kaum beachteten Sonderfall der vorgeschalteten hybriden Gesellschaft mit schweizerischer Betriebsstätte.

I. Einleitung

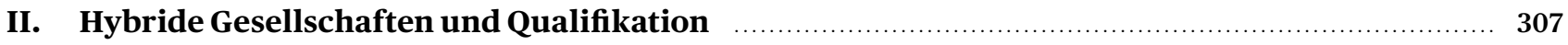

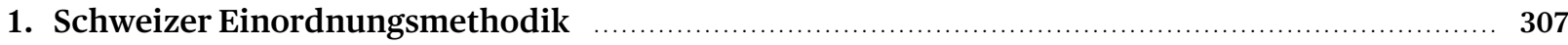

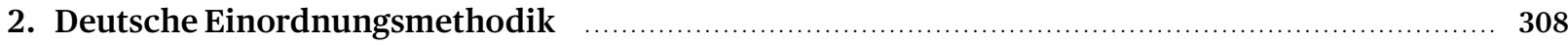

3. Sonderfall: Vorgeschaltete hybride Gesellschaft mit Betriebsstätte in der Schweiz $\quad \ldots \ldots \ldots \ldots \ldots \ldots . \ldots \ldots$

III. Vorgeschaltete hybride Gesellschaft mit schweizerischer Betriebsstätte $\ldots \ldots \ldots \ldots \ldots \ldots \ldots \ldots \ldots \ldots$

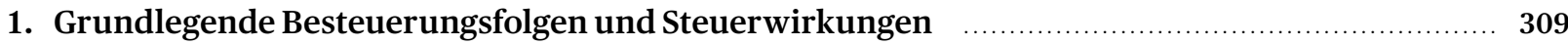

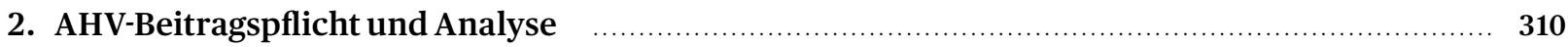

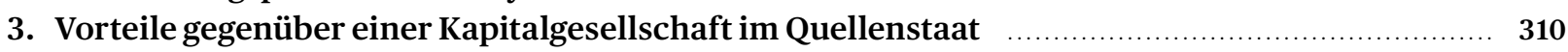

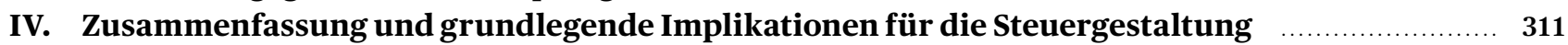

Zitiervorschlag:

THOMAS KOLLRUSS, Hybride Gesellschaften im

Verhältnis Schweiz-Deutschland, sui generis 2021, S. 305

Thomas Kollruss ist im nationalen und internationalen Steuerrecht tätig (t.kollruss@gmx.at).

URL: sui-generis.ch/194

DOI: https://doi.org/10.21257/sg.194

Dieses Werk ist lizenziert unter einer Creative Commons Namensnennung - Weitergabe unter gleichen Bedingungen 4.0 International Lizenz. 


\section{Einleitung}

1 Hybride Gesellschaften treten insbesondere bei einer grenzüberschreitenden Beteiligung an einer ausländischen Gesellschaft auf, wenn es um die Einordnung des Sachverhalts in die Besteuerung geht. So kann ein und dieselbe ausländische Gesellschaft für Zwecke der Besteuerung divergierend eingestuft werden - und zwar als Kapitalgesellschaft nach dem Steuerrecht des Sitzstaates und als transparente Personengesellschaft nach dem Steuerrecht des Ansässigkeitsstaats des Gesellschafters (hybride Gesellschaft) oder umgekehrt (reverse hybrid). Hintergrund ist, dass ausländische Gesellschaften grundsätzlich autonom nach Massgabe des jeweiligen Zivil-und Steuerrechts des Ansässigkeitsstaats des Gesellschafters in die dortige Besteuerung eingeordnet werden. Eine Übernahme der Qualifikation durch den ausländischen Sitz- bzw. Gründungsstaat des Rechtsgebildes (Souveränitätsprinzip, keine Qualifikationsverkettung) erfolgt grundsätzlich nicht.

2 Rechtsmethodisch kann die Einordnung des ausländischen Rechtsgebildes im Wesentlichen durch einen Ähnlichkeitsvergleich zu inländischen Rechtsträgern oder durch Rechtstypenvergleich erfolgen wie in der Schweiz ${ }^{1}$, Deutschland ${ }^{2}$ oder Österreich ${ }^{3}$. Andere Möglichkeiten sind die Gewährung von Einordnungswahlrechten an den Gesellschafter (check-the-box election/USA ${ }^{4}$ ) oder die Vorgabe einer Einordnungsliste durch die Finanzverwaltung auf Basis der Rechtsprechung (UK5).

3 Die abweichende steuerliche Einordnung einer ausländischen Gesellschaft durch den Sitzstaat und den Ansässigkeitsstaat des Gesellschafters bzw. den Quellenstaat

1 Vgl. Urteil des Bundesgerichts 2C 894/2013 vom 18. September 2015 (US-LLC); Schweizerische Steuerkonferenz (SSK), Praxishinweise vom 6. September 2011 zur steuerlichen Behandlung der US-amerikanischen Limited Liability Company bei den direkten Steuern; THOMAS KOLLRUSS, Rechtsvergleichende Analyse der steuerlichen Behandlung einer US-amerikanischen LLC durch die Schweiz und Deutschland, FStR 4/2013, S. $284 \mathrm{ff}$.

2 Vgl. Urteil des deutschen Bundesfinanzhofs I R34/08 vom 20. August 2008, BStBl II 2009, S. 263 (Florida-LLC); Urteil des deutschen Bundesfinanzhofs I R95/10 vom 25. Mai 2011, BStBlII 2014, S. 760 (ungarische BT); Schreiben des Bundesfinanzministeriums (BMF) vom 19. März 2004, IV B 4-S1301 USA - 22/04, BStBlI2004, S. 411 (US-LLC); fragwürdig Beschluss des Finanzgerichts München 6V 1784/20 vom 10. November 2020, Rev. BFHIB 76/20 (Vergleich LLC mit UG nach §5a GmbHG bezüglich Kapitalaufbringung).

3 Vgl. Urteil des österreichischen Verwaltungsgerichtshofs Ro 2018/ 13/0003 vom 13.Januar 2021 (Delaware Trust); GEORG KOFLER, Abschirmwirkung, Wien 2002, S. 459; GEORG KOFLER / JÜRGEN LÜDICKE/ MADELEINE SIMONEK, Hybride Personengesellschaften - Umsetzung des OECD Partnership Reports in Deutschland, Österreich und der Schweiz, IStR 10/2014, S. $349 \mathrm{ff}$.

4 Vgl. Internal Revenue Service der USA (IRS), Form 8832, Entity Classification Election, S. 2.

5 Vgl. HMRC, International Manual 180030, Foreign entity classification for UK tax purposes: List of Classifications of Foreign Entities for UK tax purposes. führt regelmässig zu einem sog. subjektiven Qualifikationskonflikt, da beide Staaten aufgrund des nationalen Steuerrechts die Einkünfte unterschiedlichen Steuersubjekten zur Besteuerung zurechnen. Dies kann zu einer Doppelbesteuerung (positiver Qualifikationskonflikt) oder zu einer Minderbesteuerung (negativer Qualifikationskonflikt) im Vergleich zu einer übereinstimmenden steuerlichen Einordung des ausländischen Rechtsgebildes führen. In einigen Fällen kann dieser subjektive Qualifikationskonflikt auch zu einer doppelten Nichtbesteuerung von Einkünften führen, so dass weder der Sitzstaat der ausländischen Gesellschaft noch der Ansässigkeitsstaat des Gesellschafters die Einkünfte besteuert. Weiterhin kann eine hybride Gesellschaft auch vorliegen, wenn eine hybride Drittstaatengesellschaft - z. B. eine US-LLCmit Betriebsstätte in der Schweiz und deutschen Gesellschaftern auftritt («Dreieckssachverhalt»). In einem solchen Fall wird die US-LLC vom Quellenstaat Schweiz grundsätzlich als juristische Person und Kapitalgesellschaft eingestuft, während der Ansässigkeitsstaat der Gesellschafter (Deutschland) von einer transparenten Personengesellschaft ausgeht und den laufenden Gewinn aus der schweizerischen Betriebsstätte im Ergebnis nach dem Doppelbesteuerungsabkommen (DBA) von der Besteuerung mit deutscher Einkommensteuer ausnimmt.

Der vorliegende Beitrag befasst sich mit hybriden Gesell- 4 schaften im Verhältnis Schweiz-Deutschland. Neben der grundlegenden Einordnung und Qualifikation von hybriden Gesellschaften in diesem Verhältnis wird aus der Gesamtmenge der hybriden Gesellschaften die vorgeschaltete hybride Gesellschaft herausgegriffen und in Bezug auf eine Direktinvestition in der Schweiz (gewerbliche Betriebsstätte) untersucht. Bei einer vorgeschalteten hybriden Gesellschaft begründet der ausländische Investor nicht unmittelbar eine Betriebsstätte in der Schweiz, sondern ist an einer hybriden ausländischen Gesellschaft beteiligt, die in der Schweiz eine Betriebsstätte unterhält (indirekte Direktinvestition). Im Idealfall sollte sich die hybride ausländische Gesellschaft für Zwecke des schweizerischen Steuerrechts als Kapitalgesellschaft bzw. Gewinnsteuersubjekt qualifizieren, so dass der Betriebsstättengewinn in der Schweiz der niedrigen Gewinnsteuer statt der höheren Einkommensteuer unterliegt. Gleichzeitig qualifiziert sich die vorgeschaltete hybride Gesellschaft im Ansässigkeitsstaat der Gesellschafter als transparente Personengesellschaft, so dass dort der Gewinn aus der schweizerischen Betriebsstätte nach dem massgeblichen DBA von der Besteuerung mit Einkommensteuer befreit wird. Vorgeschaltete hybride Gesellschaften können somit als steueroptimale rechtliche Investitionsstruktur für Direktinvestitionen von Steuerausländern in der Schweiz in Betracht kommen. ${ }^{6}$ Ein

6 Inbesondere für natürliche Personen. 
weiterer Vorteil ist, dass vorgeschaltete hybride Gesellschaften grundsätzlich nicht von der Anti-Tax-AvoidanceDirective (ATAD) betroffen sind. ${ }^{7}$ Aussersteuerliche Vorteile einer solchen Struktur liegen insbesondere in der zivilrechtlichen Haftungsbeschränkung und der gesellschaftsrechtlichen Flexibilität. Vorgeschaltete hybride Gesellschaften stellen daher eine interessante Alternative zu einer Kapitalgesellschaftsstruktur dar. Im Rahmen des Beitrags werden eine vorgeschaltete deutsche $\mathrm{GmbH}$ \& Co. KG mit Schweizer Betriebsstätte sowie eine hybride US-LLC mit Schweizer Betriebsstätte und deutschen Gesellschaftern betrachtet.

\section{Hybride Gesellschaften und Qualifikation}

\section{Schweizer Einordnungsmethodik}

5 Gemäss Art. 49 Abs. $3 \mathrm{DBG}^{8}$ werden ausländische juristische Personen sowie nach Art.11 DBG steuerpflichtige, ausländische Handelsgesellschaften und andere ausländische Personengesamtheiten ohne juristische Persönlichkeit den inländischen juristischen Personen gleichgestellt, denen sie rechtlich oder tatsächlich am ähnlichsten sind. Aus Schweizer Sicht ist zur Qualifizierung ausländischer Gesellschaften ein pragmatischer Methodenmix anzuwenden, welcher einerseits von einem Vergleich der ausländischen Gesellschaft oder Entität mit inländischen Gebilden ausgeht, dabei andererseits aber auch die steuerliche Behandlung im Inkorporationsstaat als Element mitberücksichtigt. ${ }^{9}$ Internrechtlich soll die Rechtsgrundlage für den vorgenannten Methodenmix aus Art. 49 Abs. 3 DBG in Verbindung mit dem Doppelbesteuerungsabkommen (DBA) des Sitzstaates der ausländischen Gesellschaft folgen, so das Schweizerische Bundesgericht. Weiterhin erfolgt die Qualifikation nach Schweizer Recht nicht einzig nach dem Ähnlichkeitsansatz. ${ }^{10}$ In Präzisierung der Rechtsprechung kann die ausländische Gesellschaft auch nach Schweizer Steuerrecht nicht als juristische Person qualifiziert werden, wenn das Zivilrecht des Inkorporationsstaates das ausländische Rechtsgebilde nicht als juristische Person behandelt. Massgeblich sind zudem das ausländische Gesellschaftsrecht und die darin verankerten Strukturmerkmale sowie die konkrete

7 Ein deduction no-inclusion Szenario liegt nicht vor. Der Betriebsstättengewinn wird im ausländischen Quellenstaat gerade besteuert, jedoch kann die effektive Gesamtsteuerbelastung aus der Direktinvestition massgeblich abgesenkt werden (tax rate shopping, insbesondere im ausländischen Quellenstaat).

8 Bundesgesetz über die direkte Bundessteuer vom 14. Dezember 1990 (DBG; SR 642.11).

9 Vgl. Urteil des Bundesgerichts 2C_894/2013 vom 18. September 2015 E.3.1.

10 Vgl. Urteil des Bundesgerichts 2C_894/2013 vom 18. September 2015 E.3.1.
Ausgestaltung des Gesellschaftsvertrags der ausländischen Gesellschaft. ${ }^{11}$

Jedenfalls wenn sich die ausländische Gesellschaft nach 6 dem Zivilrecht des Sitzstaates als juristische Person qualifiziert (eigene juristische Persönlichkeit), die ausländische Gesellschaft zivilrechtlich erst mit der Eintragung in ein ausländisches Register entsteht, die persönliche Haftung der Gesellschafter für Verbindlichkeiten der Gesellschaft ausgeschlossen ist und die Gewinnzuteilung einen Ausschüttungsbeschluss voraussetzt, sollte sich die ausländische Gesellschaft für Schweizer Steuerzwecke als ausländische Kapitalgesellschaft bzw. ausländische juristische Person qualifizieren. Die steuerliche Behandlung der Gesellschaft im ausländischen Sitzstaat sollte unter diesen Umständen irrelevant sein. ${ }^{12}$

Ansonsten ergibt sich auch ein Widerspruch zu Art.11 7 DBG, wonach ausländische Handelsgesellschaften und andere ausländische Personengesamtheiten ohne juristische Persönlichkeit, die aufgrund wirtschaftlicher Zugehörigkeit in der Schweiz steuerpflichtig sind, ihre Steuern nach den Bestimmungen für die juristischen Personen entrichten. ${ }^{13}$ Solche ausländischen Personengesamtheiten im Sinne des Art.11 DBG mit Betriebsstätte in der Schweiz werden somit wie juristische Personen intransparent behandelt, indem die Personengesamtheit als ein Ganzes mit Gewinnsteuer besteuert wird und nicht die Teilhaber persönlich besteuert werden. ${ }^{14}$ Dementsprechend und nach einem Erst-recht-Schluss - argumentum a minoriad

11 Vgl. Urteil des Bundesgerichts 2C_894/2013 vom 18. September 2015 E.3.3 ff.; SSK, Praxishinweis US-LLC (Fn.1), S. 2 f.

12 Insgesamt ergibt sich ein Bruch bzw. eine (methodische) Zweiteilung innerhalb der Qualifikation ausländischer Gesellschaften dahingehend, dass die Besteuerung der ausländischen Gesellschaft in ihrem Sitzstaat für die steuerliche Einordnung in der Schweiz faktisch irrelevant ist, wenn die ausländische Gesellschaft in der Schweiz aufgrund wirtschaftlicher Zugehörigkeit steuerpflichtig ist. Der Regelungsbefehl des Art.11 DBG lässt eine Berücksichtigung der Besteuerung des ausländischen Gebildes im ausländischen Sitzstaat als Einordnungskriterium nicht zu. Dies würde auch die gesetzliche Regelung und angeordnete Rechtsfolge in ihr Gegenteil verkehren. In der Literatur wird daher auch zutreffend von einer Sonderregelung gesprochen. Vgl. hierzu KOFLER/LÜDICKE/SIMONEK (Fn. 3), S. 351; PETER BRÜLISAUER / MARCEL R. KRIESI, Internationale Personenunternehmen im Einkommens- und Gewinnsteuerrecht der Schweiz (1. Teil), FStR 2007, S. 272 und 275.

13 Dies gilt unabhängig von der steuerlichen Behandlung der ausländischen Handelsgesellschaft bzw. der ausländischen Personengesamtheit ohne juristische Persönlichkeit in ihrem ausländischen Sitzstaat. Ansonsten würde der Gesetzesbefehl des Art.11 DBG faktisch leerlaufen, wenn bei ausländischen Gesellschaften i.S. des Art.11 DBG zusätzlich die steuerliche Behandlung im ausländischen Sitzstaat heranzuziehen wäre. Folglich dürfte Art. 49 Abs. 3 DBG und damit die Mitberücksichtigung der steuerlichen Behandlung des Rechtgebildes im ausländischen Sitzstaat bei der Einordnung des ausländischen Rechtsgebildes in das Schweizer Steuerrecht nur eine Teilmenge ausländischer Gesellschaften betreffen und zwar solche, die in der Schweiz nicht steuerpflichtig sind, also insbesondere nicht aufgrund einer wirtschaftlichen Zugehörigkeit. Vgl. auch KOFLER/ LÜDICKE/SIMONEK (Fn.3), S.351.

14 Vgl. Urteil des Bundesgerichts 2C_894/2013 vom 18. September 2015 E. 2.5.1. 
maius - ist eine US-LLC, die nach US-Recht eigene Rechtspersönlichkeit besitzt und sich nach dortigem Zivilrecht als eigenständige juristische Person qualifiziert ${ }^{15}$, und die eine Betriebsstätte in der Schweiz unterhält und damit aufgrund wirtschaftlicher Zugehörigkeit in der Schweiz steuerpflichtig ist, erst recht für schweizerische Steuerzwecke als intransparente juristische Person, sprich ausländische Kapitalgesellschaft zu besteuern. ${ }^{16}$

8 Im Ergebnis wird damit eine deutsche GmbH \& Co. KG (ausländische Handelsgesellschaft) mit Betriebsstätte in der Schweiz wie eine ausländische juristische Person mit Gewinnsteuer besteuert. ${ }^{17}$ Eine US-LLC mit Betriebsstätte in der Schweiz, die aufgrund wirtschaftlicher Zugehörigkeit in der Schweiz steuerpflichtig ist, wird ebenfalls nach den Bestimmungen für juristische Personen besteuert und unterliegt selbst der Gewinnsteuer in der Schweiz. ${ }^{18}$ In der Schweiz ist somit allein die US-LLC Steuersubjekt hinsichtlich der Besteuerung der Gewinne aus der schweizerischen Betriebsstätte und nicht ihr ausländischer Gesellschafter. Die vorstehenden Ausführungen gelten unabhängig von der steuerlichen Behandlung der deutschen GmbH \& Co. KG ${ }^{19}$ sowie der US-LLC ${ }^{20}$ in ihrem Sitzstaat sowie im ausländischen Ansässigkeitsstaat der Gesellschafter.

\section{Deutsche Einordnungsmethodik}

9 Für Zwecke der deutschen Ertragsbesteuerung ist ein zweistufiger Rechtstypenvergleich durchzuführen bezüglich der Einordnung einer ausländischen Gesellschaft

15 Vgl. Florida revised LLC Act, chapter 605.0108 (1); WY Stat \$17-29104 (a), Wyoming LLC Act 2015; MARTIN M. SHENKMAN / SAMUEL WEINER / IVAN TABACK, Starting a Limited Liability Company, 2. Aufl., Hoboken 2003, S. 4, 64; Urteil des deutschen Bundesfinanzhofs IR 34/08 vom 20. August 2008, BStBl II 2009, S. 263; SSK, Praxishinweis US-LLC (Fn.1) mit Hinweis auf §18-201b des Delaware Limited Liability Company Act.

16 Vgl. SSK, Praxishinweis US-LLC (Fn. 1), S. 3. Dem steht nicht das Urtei des Bundesgerichts 2C_894/2013 vom 18. September 2015 entgegen, da es hier explizit um eine US-LLC ohne Steuerpflicht in der Schweiz aufgrund wirtschaftlicher Zugehörigkeit nach Art. 51 Abs.1 lit.b sowie Abs. 2 DBG ging.

17 Vgl. auch MICHAEL LANG / MARKUS REICH, Personengesellschaften im Verhältnis Deutschland-Österreich-Schweiz, Der Schweizer Treuhänder 10/2006, S. 764 f.; Urteil des Bundesgerichts 2C_894/2013 vom 18. September 2015 E. 2.5.1.

18 Vgl. SSK, Praxishinweis US-LLC (Fn. 1), S. 3.

19 Die deutsche GmbH \& Co. KG wird in Deutschland als transparente Personengesellschaft besteuert, sofern nicht die Option zur Körperschaftsteuer gewählt wird (\$1a KStG). Einkommensteuersubjekte und Steuerpflichtige sind die Gesellschafter mit ihrem Gewinnanteil aus der KG ( $\$ 15$ Abs. 1 Nr. 2 Einkommensteuergesetz [Deutschland; EStG-D]). Eine Einmann-GmbH \& Co. KG ist möglich, indem die natürliche Person zu 100\% als Kommanditistin an der KG beteiligt ist und $100 \%$ der Anteile an der Komplementär-GmbH hält, die nicht am Gewinn und Vermögen der KG beteiligt ist.

20 Eine Single-Member LLC ist grundsätzlich möglich. Vgl. Website des Internal Revenue Service (IRS) über Single-Member LLC. bzw. der Beteiligung an einer ausländischen Gesellschaft. ${ }^{21}$ Massgeblich sind im Grundsatz die - aus der finanzgerichtlichen Rechtsprechung abgeleiteten - fünf Kriterien: Geschäftsführung und Vertretung, beschränkte Haftung, freie Übertragbarkeit der Anteile, Gewinnzuteilung und Kapitalaufbringung. ${ }^{22}$ Eine Qualifikationsverkettung bzw. die Übernahme der ausländischen Qualifikation, insbesondere durch den Sitzstaat des ausländischen Rechtsgebildes, und eine Berücksichtigung der steuerlichen Behandlung des ausländischen Rechtsgebildes im Ausland erfolgen nicht bei der Einordnung eines ausländischen Rechtsgebildes in das deutsche Ertragsteuerrecht. ${ }^{23}$ Anschliessend wird die konkrete Ausprägung dieser Kriterien bei der betreffenden ausländischen Gesellschaft nach Massgabe des ausländischen Gesellschaftsrechts einschliesslich der Regelungen im Gesellschaftsvertrag und Satzung jeweils festgestellt. Schliesslich wird für jedes der vorgenannten Kriterien jeweils festgestellt, ob die ausländische Gesellschaft nach Massgabe der tatsächlichen Ausprägung des Kriteriums eher einer deutschen Personengesellschaft oder einer Körperschaft/Kapitalgesellschaft entspricht. Die ausländische Gesellschaft entspricht dann einer deutschen Körperschaft, wenn die Mehrzahl der vorgenannten Kriterien für eine Körperschaft ausfallen (Gesamtbildbetrachtung), ansonsten ist die ausländische Gesellschaft als transparente Personengesellschaft oder als Betriebsstätte bei einem Alleingesellschafter zu qualifizieren. Nach der Rechtsprechung muss das Vorliegen einer ausländischen Körperschaft im Rahmen des Rechtstypenvergleichs positiv festgestellt werden. Da das Gesellschaftsrecht der US-Bundesstaaten im Hinblick auf die LLC grundsätzlich sehr flexibel und auch dispositiv ist, kann durch entsprechende Ausgestaltung des Gesellschaftsvertrags eine Qualifikation der US-LLC als transparente Personengesellschaft für deutsche Ertragsteuerzwecke erreicht werden. ${ }^{24}$

\section{Sonderfall: Vorgeschaltete hybride Gesellschaft mit Betriebsstätte in der Schweiz}

Eine US-LLC mit Betriebsstätte in der Schweiz, die in 10 der Schweiz als juristische Person mit Gewinnsteuer

21 Vgl. allgemein zum deutschen Rechtskreis: MARIA G. MARQUARDSEN, Hybride Gesellschaften im Internationalen Steuerrecht der Bundesrepublik Deutschland, 2019.

22 Vgl. Urteil des deutschen Bundesfinanzhofs I R 34/08 vom 20. August 2008, BStBlII 2009, S. 263; Urteil des deutschen Bundesfinanzhofs I R 95/10 vom 25. Mai 2011, BStBl II 2014, S. 760; Schreiben des deutschen Bundesfinanzministeriums vom 19. März 2004, IV B 4-S1301 USA-22/04, BStBlI 2004, S. 411.

23 Vgl. ständige Rechtsprechung, Urteil des deutschen Bundesfinanz hofs I R 95/10 vom 25. Mai 2011, BStBl II 2014, S. 760; Urteil des deutschen Bundesfinanzhofs I R 67/12 vom 13. November 2013, BStBl II 2014, S.172.

24 Vgl.Schreiben des deutschen Bundesfinanzministeriums vom 19.März 2004, IVB 4-S1301 USA - 22/04, BStBlI 2004, S. 411 (zur US-LLC). 
besteuert wird, kann in Deutschland als transparente Personengesellschaft behandelt werden. Weiterhin wird eine deutsche GmbH \& Co. KG mit Betriebsstätte in der Schweiz dort selbst mit Gewinnsteuer besteuert, während Deutschland diese als transparente Personengesellschaft behandelt und die Gesellschafter mit ihrem Gewinnanteil besteuert. Somit können im Verhältnis Schweiz-Deutschland hybride Gesellschaften auftreten und auch genutzt werden. Insbesondere für in Deutschland ansässige natürliche Personen kann es sich zivil- und steuerrechtlich anbieten, eine Direktinvestition in der Schweiz in Form einer Betriebsstätte nicht unmittelbar zu tätigen, sondern als Gesellschafter einer vorgeschalteten hybriden Gesellschaft, welche die Betriebsstätte in der Schweiz unterhält (indirekte Direktinvestition).

\section{Vorgeschaltete hybride Gesellschaft mit schweizerischer Betriebsstätte}

\section{Grundlegende Besteuerungsfolgen und Steuerwirkungen}

11 Ein in Deutschland ansässiger ${ }^{25}$ Investor (natürliche Person) kann zunächst direkt eine (gewerbliche) Betriebsstätte ${ }^{26}$ in der Schweiz begründen. Dies hat zur Folge, dass in der Schweiz die Besteuerungsregelungen für natürliche Personen zur Anwendung kommen. Im Rahmen der beschränkten Steuerpflicht (wirtschaftliche Zugehörigkeit) unterliegt der laufende Gewinn aus der Betriebsstätte in der Schweiz zunächst der schweizerischen Einkommenssteuer. ${ }^{27}$ Entsprechendes gilt für etwaige Gewinne aus der Veräusserung der schweizerischen Betriebsstätte (aperiodische Besteuerung). ${ }^{28}$ Gemäss Art. 7 Abs. 2 DBG einschliesslich der Regelungen der Kantone und Gemeinden ist die Einkommenssteuer mindestens zu dem Steuersatz zu entrichten, der dem in der Schweiz erzielten Einkommen entspricht. ${ }^{29}$ Für das Steuerjahr 2021 ist ein maximaler effektiver Einkommenssteuersatz von 22,38\% (Kanton Zug) bis 44,75\% (Genf) massgeblich,

25 Vgl. Schreiben des deutschen Bundesfinanzministeriums vom 19. März 2004, IV B 4-S1301 USA - 22/04, BStBlI 2004, S. 411.

26 Beispielsweise Produktion von Waren. Es wird unterstellt, dass eine aktiv tätige Betriebsstätte begründet wird, die Unternehmensgewinne im Sinne des Art. 7 DBA Schweiz-Deutschland generiert. Die Regelungen der deutschen Hinzurechnungsbesteuerung sind daher nicht einschlägig ( $\$ 20$ Abs. 2 deutsches Gesetz über die Besteuerung bei Auslandsbeziehungen vom 8. September 1972, AStG-D).

27 Vgl. Art. 4 Abs. 1 lit. b, Abs. 2, Art. 6 Abs. 2 DBG; Art. 7 Abs. 1 und 2 DBA Schweiz-Deutschland.

28 Vgl. Art. 18 Abs. 2 DBG; Art. 13 Abs. 1 und 2 DBA Schweiz-Deutschland.

29 Der Spitzensteuersatz bei der progressiven direkten Bundessteuer (Einkommenssteuer) natürlicher Personen beträgt 11,5\%. Vgl. Art.36 DBG. wobei der durchschnittliche effektive Einkommenssteuersatz über alle Kantone hinweg $33,73 \%$ beträgt. ${ }^{30}$

Hinzu kommen einkommensabhängige AHV-Beiträge als 12 Selbständigerwerbender in der Schweiz; in der Spitze 10\% des jährlichen Erwerbseinkommens. ${ }^{31}$ Hingegen beläuft sich der maximale effektive Gewinnsteuersatz 2021 bei einem schweizerischen Betriebsstättengewinn in gleicher Höhe auf lediglich zwischen 11,85\% (Kanton Zug) und 21,04\% (Kanton Bern). ${ }^{32}$ Für eine natürliche Person als ausländischer Investor in der Schweiz könnte es sich daher als Alternative anbieten, eine schweizerische Betriebsstätte nicht direkt, sondern über eine vorgeschaltete hybride Gesellschaft zu unterhalten, beispielsweise über eine deutsche $\mathrm{GmbH} \&$ Co. KG. Die Steuerbelastung im Quellenstaat bzw. Betriebsstättenstaat Schweiz ist geringer. Die Besteuerung mit Einkommenssteuer im Ansässigkeitsstaat Deutschland ändert sich nicht; es bleibt dort grundsätzlich bei der Steuerfreistellung des Betriebsstättengewinns aus der Schweiz. ${ }^{33}$ Gleichzeitig lässt sich durch die Wahl der Rechtsform der GmbH \& Co. KG zivilrechtlich eine Begrenzung der Haftung erreichen. Grafisch lässt sich die rechtliche Investitionsalternative (vorgeschaltete hybride Gesellschaft in Form einer deutschen GmbH \& Co. KG) wie folgt abbilden:

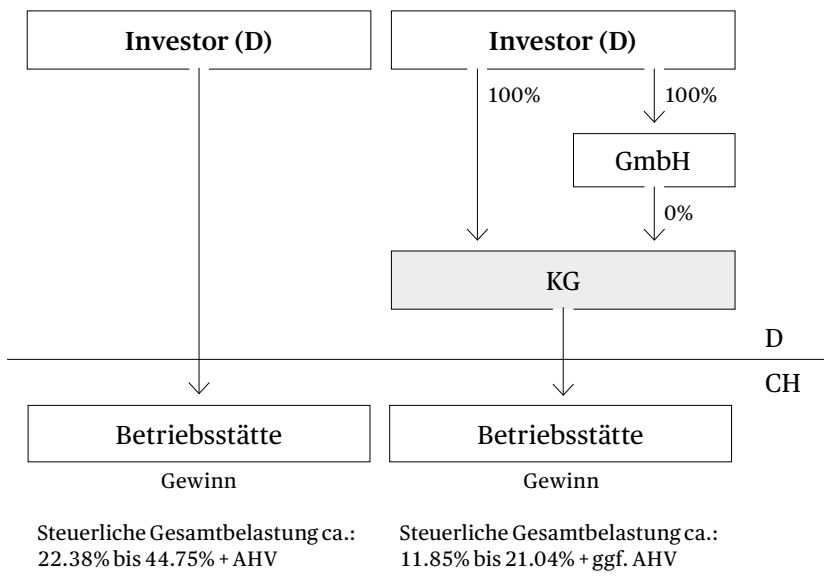

Die Nutzung der vorgeschalteten hybriden Gesellschaft 13 führt zu einer massgeblich niedrigeren Gesamtsteuerbelastung als bei direkter Unterhaltung der Schweizer Betriebsstätte. Im Ergebnis lässt sich durch die vorgeschaltete hybride Gesellschaft bei der natürlichen Personen (D) eine finale Gesamtsteuerbelastung in Höhe der

30 Vgl. KPMG, Clarity on Swiss Taxes, April 2021, S. 21 (maximal effektiver Einkommenssteuersatz Bund/Kanton/Gemeinde für den jeweiligen Hauptort).

31 Vgl. AHV, Beitragsskala für Selbständigerwerbende 2021. Vgl. allgemein zur AHV-Pflicht LANG/REICH (Fn. 17), S.766. Vgl. Art. 33 Abs. 1 lit. d DBG zur Abzugsfähigkeit der AHV-Beiträge.

32 Vgl. KPMG (Fn.30), S.8f. Der durchschnittliche Gewinnsteuersatz über alle Kantone beträgt im Jahr 202114,87\%.

33 Vgl. Art.7 Abs. 1 und 2 sowie Art. 24 Abs. 1 Nr. 1a DBA Schweiz-Deutschland: Freistellung unter Progressionsvorbehalt im Rahmen der deutschen Besteuerung mit Einkommenssteuer. 
niedrigen Schweizer Gewinnsteuer erreichen in Bezug auf Schweizer Betriebsstättengewinne. Die Steuerfreistellung des schweizerischen Betriebsstättengewinns in Deutschland nach Art. 24 Abs. 1 Nr.1a DBA SchweizDeutschland wird nicht dadurch ausgeschlossen, dass die Schweiz die deutsche KG wie eine juristische Person mit Gewinnsteuer besteuert (Art.11 DBG). Erstens enthält das DBA Schweiz-Deutschland ${ }^{34}$ diesbezüglich keine Subject-to-tax Klauseln (Besteuerungsrückfall) und Switchover Klauseln (Methodenwechsel zur Anrechnungsmethode). ${ }^{35}$ Zweitens wird der Betriebsstättengewinn in Übereinstimmung mit Art. 7 DBA Schweiz-Deutschland in der Schweiz besteuert. Drittens sind unilaterale switchover Regelungen nach deutschem Steuerrecht ${ }^{36}$ nicht einschlägig, da der Betriebsstättengewinn im Quellenstaat Schweiz gerade vollumfänglich besteuert wird und diese Regelungen keine strenge Steuersubjektidentität ${ }^{37}$ verlangen. Viertens liegt auch keine hybride Gestaltung nach $\mathrm{ATAD}^{38}$ vor, da der Betriebsstättengewinn im Quellenstaat Schweiz gerade vollständig besteuert wird. OECD Pillar 2-globale Mindeststeuer - findet auf Einkommensteuersubjekte grundsätzlich keine Anwendung (natürliche Person D). ${ }^{39}$

34 Art. 4 Abs.11DBA Schweiz-Deutschland ist nicht einschlägig, da vor liegend die Einkünfte aus der schweizerischen Betriebsstätte übereinstimmend durch den Quellenstaat Schweiz und den Ansässigkeitsstaat Deutschland jeweils dem beschränkt steuerpflichtigen deutschen KG-Gesellschafter (als Einkünfteerzielungssubjekt) zugerechnet werden. Art.11 und Art. 49 Abs.3 DBG ändern hieran nichts. Nach diesen Regelungen erfolgt lediglich die Besteuerung der be schränkt steuerpflichtigen Einkünfte des Gesellschafters (wirtschaftliche Zugehörigkeit), die diesem anteilsmässig zugerechnet werden (Art.10 Abs.1 DBG), auf der «Ebene der Personengesellschaft» mit Gewinnsteuer. Art.11 DBG setzt vorgelagert eine Steuerpflicht der im Rahmen der ausländischen Personengesellschaft erzielten Einkünfte, die selbst kein Steuersubjekt ist, voraus, nämlich die Steuerpflicht qua wirtschaftlicher Zugehörigkeit der Gesellschafter der ausländischen Personengesellschaft mit den Einkünften aus der ausländischen Personengesellschaft in der Schweiz, insbesondere nach Art. 6 Abs. 2 und Art. 4 Abs. 1 lit. b DBG. Demzufolge ist dann auch mit der Besteuerung der schweizerischen Betriebsstätteneinkünfte nach Art.11 DBG die Besteuerung des beschränkt steuerpflichtigen deutschen KG-Gesellschafters in der Schweiz im Rahmen seiner wirtschaftlichen Zugehörigkeit erfolgt und abgeschlossen, eine weitere Besteuerung dieser Person erfolgt nicht (Einmalbesteuerung entsprechend Art. 10 Abs. 1 DBG). Vgl. allgemein zu Art. 4 Abs. 11 DBA Schweiz-Deutschland HEIKO KUBAILE, in: Flick/Wassermeyer/ Kempermann(Hrsg.), Doppelbesteuerungsabkommen DeutschlandSchweiz, Lfg. 7/2018, Bonn 1981, Art. 4 N233ff. Nach dem DBA Schweiz Deutschland sind Personengesellschaften abkommensrechtlich grundsätzlich keine Personen (abkommensautonome Auslegung).

35 Vgl. auch NILS HÄCK, in: Flick/Wassermeyer/Kempermann(Hrsg.), Doppelbesteuerungsabkommen Deutschland-Schweiz, Lfg. 12/2015, Bonn 1981, Art. 24 N 4, 4.1, 9 und 20.

36 \$od Abs. 9 Nr. 1 bis 3 EStG-D.

37 Vgl. hierzu THOMAS KOLLRUSS, Ungeklärte ertragsteuerliche Fragen hybrider ausländischer Gesellschaften, StuW 2010, S. 383 ff.; DIETMAR GOSCH, in: Kirchhof/Seer (Hrsg.), Einkommensteuergesetz, Kommentar, 20. Aufl., Köln 2021, § 50d Abs. 9 EStG N 41ab.

38 Vgl.EU-Richtlinie 2017/952 des Rates vom 29. Mai 2017 (Anti-Tax Avoidance Directive, ATAD), Art. 9 (Hybride Gestaltungen), Art. 9 Abs. 5 , Art. 9a (Umgekehrt hybride Gestaltungen), Art. 9b ATAD.

39 Im Übrigen wäre der Heraufschleusungseffekt auf 15\% Steuergesamtbelastung bei einer Gewinnsteuerbelastung (Regelbesteuerung) von

\section{AHV-Beitragspflicht und Analyse}

Die von einer im Ausland ansässigen natürlichen Per- 14 son (D) über eine deutsche GmbH \& Co. KG bezogenen Gewinne aus einer Schweizer Betriebsstätte unterliegen grundsätzlich der AHV-Beitragspflicht. Der deutsche Gesellschafter qualifiziert sich im Rahmen seines Gewinnanteils als Selbständigerwerbender in der Schweiz. ${ }^{40}$ Unterhält die deutsche GmbH \& Co. KG neben ihrer schweizerischen Betriebsstätte noch weitere, nicht unwesentliche gewerbliche bzw. unternehmerische Betriebsstätten in Deutschland, erfolgt eine Unterstellung nach dem Wohnsitzprinzip (Gesellschafter), so dass grundsätzlich die AHV-Beitragspflicht in der Schweiz entfällt. ${ }^{41}$ In solchen Konstellationen ist die Rechtsform der deutschen $\mathrm{GmbH}$ \& Co. KG hinsichtlich einer etwaigen AHV-Beitragspflicht mindestens äquivalent, wenn nicht sogar vorteilhaft gegenüber der Wahl einer schweizerischen Kapitalgesellschaft ceteris paribus als Rechtsform für die Direktinvestition (Schweizer Betriebsstätte). ${ }^{42}$ Es kann sich somit anbieten, neben der schweizerischen Betriebsstätte weitere deutsche gewerbliche Betriebsstätten in der $\mathrm{GmbH}$ \& Co. KG zu bündeln. ${ }^{43}$ Im Übrigen stellt eine etwaige AHVBeitragspflicht bei vorgeschalteter GmbH \& Co. KG keinen Nachteil zum Fall der Direktunterhaltung der schweizerischen Betriebsstätte durch die im Ausland ansässige natürliche Person dar, da auch in dieser Konstellation AHV-Beiträge identisch zu prüfen sind.

\section{Vorteile gegenüber einer Kapitalgesellschaft im Quellenstaat}

Gegenüber einer schweizerischen Kapitalgesellschaft 15 hat die rechtliche Investitionsstruktur über eine vorgeschaltete deutsche GmbH \& Co. KG den zusätzlichen Vorteil, dass (laufende) Verluste aus der schweizerischen Betriebsstätte in Deutschland auf der Ebene der Gesellschafter (Kommanditist, natürliche Person) grundsätzlich im

$11,85 \%$ in der Schweiz relativ gering. Vgl. zu Pillar 2: OECD, Statement on a Two-Pillar Solution to Address the Tax Challenges Arising From the Digitalisation of the Economy, 1. July 2021.

40 Vgl. ORLANDO RABAGLIO, Personengesellschaften im Fadenkreuz der AHV, Trex 5/2015, S. 294 ff.; WERNER BEILSTEIN / CORINNE SCAGNET, Vollumfängliche AHV-Beitragspflicht für die deutsche $\mathrm{GmbH}$ \& Co. KG, Der Schweizer Treuhänder 10/2008, S. 750 ff.; LANG/REICH (Fn.17), S. 766.

41 Vgl. auch Bundesamt für Sozialversicherungen (BSV), Wegleitung über die Versicherungspflicht in der AHV/IV (WVP), Stand 1.Januar 2021, 30871/21, 2042.11/18 und 2042.21/17; LANG/REICH (Fn. 17), S. 766.

42 Zu berücksichtigen wären auch Geschäftsführergehälter, damit in Zusammenhang stehende AHV-Beiträge und die Besteuerung der Vergütungen einschliesslich der abkommensrechtlichen Behandlung in den beiden Vertragsstaaten (u.a. Art. 15 Abs. 4 DBA SchweizDeutschland).

43 Nach deutschem Umwandlungssteuerrecht (Umstrukturierung) können deutsche Betriebsstätten jederzeit steuerneutral in die GmbH \& Co. KG gegen Gewährung von Mitunternehmeranteilen eingebracht werden ( $\$ 24$ deutsches Umwandlungssteuergesetz vom 7. Dezember 2006 [UmwStG-D]). 
Wege des negativen Progressionsvorbehalts ${ }^{44}$ berücksichtigt werden können. Bei einer schweizerischen Kapitalgesellschaft sind Verluste grundsätzlich in dieser eingeschlossen. Ein weiterer Vorteil der vorgeschalteten deutschen $\mathrm{GmbH} \&$ Co. KG gegenüber einer schweizerischen Kapitalgesellschaft ist eine steueroptimale $\mathrm{Ge}$ winnrepatriierung. So fällt keine schweizerische Verrechnungssteuer ${ }^{45}$ auf Gewinnentnahmen aus der deutschen $\mathrm{GmbH} \& \mathrm{Co} . \mathrm{KG} \mathrm{an}^{46}$, die ceteris paribus Dividendenzahlungen einer schweizerischen Kapitalgesellschaft entsprechen würden. Weiterhin unterbleibt eine Besteuerung von Dividenden wie bei Gewinnausschüttung einer schweizerischen Kapitalgesellschaft auf der Ebene der KG-Gesellschafter in Deutschland, da vorliegend vollständig steuerneutrale Gewinnentnahmen aus einer transparenten Personengesellschaft gegeben sind. ${ }^{47}$ Auch im Veräusserungsfall (Exit) dürfte die Investitionsstruktur mit einer deutschen GmbH \& Co. KG gegenüber einer Beteiligung an einer schweizerischen Kapitalgesellschaft unter sonst gleichen Bedingungen steuerlich von Vorteil sein. Etwaige Veräusserungsgewinne, beispielsweise durch Veräusserung der Kommanditanteile oder der schweizerischen Betriebsstätte unterliegen im Ergebnis einer Steuerbelastung auf Basis der niedrigen schweizerischen

44 Bei der schweizerischen Betriebsstätte handelt es sich um eine aktiv tätige gewerbliche Betriebsstätte im Sinne des $\$ 2$ a Abs. 2 S. 1, Abs. 1 Nr. 2 EStG-D. Restriktionen könnte der negative Progressionsvorbehalt allenfalls in den Grenzen des $\$ 15$ a EStG-D erfahren (Verluste bei beschränkter Haftung). Soweit durch Verluste ein negatives Kapitalkonto des Kommanditisten in der KG entsteht oder sich erhöht (verrechenbarer Verlust), wird auch der negative Progressionsvorbehalt (temporär) gesperrt. Vgl. auch Urteil des deutschen Bundesfinanzhofs IR 182/87 vom 17. Oktober 1990, BStBl II 1991, S.136; Urteil des deutschen Bundesfinanzhofs IV R 58/00 vom 16. Mai 2002, BStBlII 2002, S. 748.

45 Unter Berücksichtigung von Art.10 Abs. 2 lit. cDBA Schweiz-Deutsch land mindestens $15 \%$ der Bruttodividende (im anderen Vertragsstaat ansässige natürliche Person als Dividendenempfänger).

46 Internrechtlich qualifiziert sich die deutsche GmbH \& Co. KG nicht als juristische Person, so dass schon nach dieser Massgabe kein Vermögensertrag im Sinne von Art. 20 DBG vorliegt. Zudem mangelt es an einer persönlichen steuerlichen Zugehörigkeit der deutschen GmbH \& Co. KG in der Schweiz, da sich weder «Satzungssitz» noch der Ort der tatsächlichen Geschäftsleitung in der Schweiz befinden (Art.50 DBG), insbesondere für Zwecke der Erhebung von Verrechnungssteuer; ein erforderlicher Inländerstatus gemäss Art. 4 Abs. 1 lit.b, Art. 9 Abs. 1 Bundesgesetz über die Verrechnungssteuer vom 13. Oktober 1965 (Verrechnungssteuergesetz, VStG; SR 642.21) dürfte nicht gegeben sein. Überdies fehlt eine steuerbare Leistung im Sinne der Verrechnungssteuer nach Art. 4 Abs. 1 lit. b VStG (Gewinnanteile/ Erträge aus Aktien, Stammanteile an $\mathrm{GmbH}$, Genossenschaftsanteile, etc.). Schliesslich kann gemäss Art. 21 DBA Schweiz-Deutschland im Zusammenhang mit der Gewinnrepatriierung bzw. mit Entnahmen aus der KG und Entnahmen aus der schweizerischen Betriebsstätte der KG keine Besteuerung in der Schweiz erfolgen. Die deutsche KG ist abkommensrechtlich nicht in der Schweiz ansässig.

47 Der Nachversteuerungseffekt mit Einkommensteuer von Dividenden aus Kapitalgesellschaften auf der Ebene von natürlichen Personen als Gesellschafter, die in Deutschland ansässig sind, beträgt in der Spitze ca. $27 \%$ bezogen auf die gezahlte Bruttodividende. Die Investitionsstruktur mit einer vorgeschalteten deutschen $\mathrm{GmbH}$ \& Co. KG kommt daher auch für deutsche Kapitalgesellschaften in Betracht. Die pauschale Schachtelstrafe von $5 \%$ bei Dividendenbezügen und Anteilsveräusserungsgewinnen lässt sich vermeiden.
Gewinnsteuer. ${ }^{48}$ Gewinne aus der Veräusserung von Anteilen an schweizerischen Kapitalgesellschaften werden hingegen im Ansässigkeitsstaat des Veräusserers (Deutschland) mit Einkommensteuer besteuert (Art.13 Abs. 3 DBA Schweiz-Deutschland). ${ }^{49}$

\section{Zusammenfassung und grundlegende Implikationen für die Steuergestaltung}

Aus der Menge der hybriden Gesellschaften stellen vor- 16 geschaltete hybride Gesellschaften eine grundsätzlich interessante Rechtsformgestaltung für grenzüberschreitende Direktinvestitionen dar. Dies gilt insbesondere für natürliche Personen, die in eine ausländische Betriebsstätte investieren wollen. Die Besonderheit und der Vorteil der Rechtsformgestaltung der vorgeschalteten hybriden Gesellschaft liegt insbesondere darin, dass sie die Vorteile von ausländischen Kapitalgesellschaften (niedrige Gewinnsteuerbelastung) und Haftungsbegrenzung mit den Vorteilen von transparenten Personengesellschaften bzw. Betriebsstätten verbindet, nämlich steuerliche Freistellung der ausländischen Betriebsstättengewinne im Ansässigkeitsstaat des Investors bei gleichzeitig flexibler und steueroptimaler Gewinnrepatriierung und mögliche steuerliche Berücksichtigung von (laufenden) Verlusten. Die Besonderheit von vorgeschalteten hybriden Gesellschaften besteht darin, dass sie sich nicht im ausländischen Quellenstaat wie herkömmliche hybride Gesellschaften befinden, sondern im Ansässigkeitsstaat des Investors oder in einem Drittstaat und vom ausländischen Quellenstaat als Gewinnsteuersubjekt bzw. Körperschaftsteuersubjekt behandelt werden, während andere involvierte Staaten von einer transparenten Gesellschaft

48 Diese Veräusserungstatbestände werden im Ergebnis wie die (anteilige) Veräusserung der Wirtschaftsgüter der schweizerischen Betriebsstätte behandelt, wobei der Gewinn auch im Rahmen der schweizerischen Betriebsstättenbilanz anfällt (direkte Methode). Vgl. auch Authorised OECD Approach (AOA). Demzufolge unterliegen etwaige Veräusserungsgewinne in Deutschland grundsätzlich der abkommensrechtlichen Freistellung unter Progressionsvorbehalt nach Art. 7, Art.13 Abs.1 und 2 sowie Art. 24 Abs.1 lit. a Satz1 und 2 DBA Schweiz-Deutschland. Für Zwecke der schweizerischen Besteuerung wird das Anfallen des Veräusserungsgewinns in Form von Gewinnen aus der schweizerischen Betriebsstätte der deutschen $\mathrm{GmbH} \&$ Co. KG nicht von Art.11, Art. 49 Abs. 3 DBG ausgeschlossen, da diese Regelungen erst nachgelagert eingreifen und das Erzielen von Einkünften durch die ausländische Personenhandelsgesellschaft in Bezug auf schweizerische Betriebsstättengewinne voraussetzen (Personengesellschaft als Subjekt der Gewinnerzielung, Gewinnermittlung und Einkünftequalifikation). Erst hieran setzen Art.11 und Art. 49 Abs. 3 DBG an. Gewinne aus der Veräusserung von Kapitalgesellschaftsbeteiligungen, insbesondere im Sinne des Art. 20 und Art. 20a DBG, sind nicht gegeben (keine Beteiligung an Kapitalgesellschaft oder Genossenschaft). Veräusserungsverluste können in Deutschland bei natürlichen Personen grundsätzlich im Wege des negativen Progressionsvorbehalts geltend gemacht werden.

49 Unter Berücksichtigung des Teileinkünfteverfahrens (40\%-ige Steuerbefreiung) bzw. der Abgeltungsteuer. 
ausgehen. Durch Nutzung vorgeschalteter hybrider Gesellschaften für ausländische Betriebsstätteninvestitionen können natürliche Personen faktisch eine Besteuerung auf Basis des niedrigen Gewinnsteuersatzes im ausländischen Quellenstaat erreichen, statt dort der wesentlich höheren Einkommenssteuer zu unterliegen. Gleichzeitig können sie eine belastende Dividendenbesteuerung im Quellen- und insbesondere Ansässigkeitsstaat vermeiden im Vergleich zur Wahl einer ausländischen Kapitalgesellschaft.

Im Verhältnis Schweiz-Deutschland kommt die deutsche GmbH\& Co. KG mit Betriebsstätte in der Schweiz als rechtliche Investitionsstruktur für eine Direktinvestition in Betracht. Dies gilt insbesondere für in Deutschland ansässige natürliche Personen. Eine weitere Möglichkeit besteht diesbezüglich in der Nutzung einer hybriden USLLC mit Betriebsstätte in der Schweiz als vorgeschaltete hybride Gesellschaft. Auch hier lässt sich im Ergebnis eine steuerliche Gesamtbelastung auf Basis der Schweizer Gewinnsteuer erreichen bei Steuerfreistellung der schweizerischen Betriebsstättengewinne auf der Ebene der deutschen Gesellschafter der US-LLC. Da es sich bei der US-LLC aus Sicht der Schweiz um eine juristische Person handelt, dürfte eine etwaige AHV-Beitragspflicht grundsätzlich nicht bestehen. Der vorliegende Beitrag konnte somit aufzeigen, dass auch im Staatenverhältnis Schweiz-Deutschland vielfältige Steuerplanungsmöglichkeiten durch Nutzung spezieller hybrider Gesellschaften bestehen, hier vorgeschaltete hybride Gesellschaften. Vorgeschaltete hybride Gesellschaften stellen eine andere Art und Möglichkeit der Steuerplanung wie mit herkömmlichen hybriden Gesellschaften dar und werden grundsätzlich nicht von den ATAD-Regelungen tangiert. Gleichzeitig können sie einen massgeblichen Beitrag zur Steuerplanung leisten, insbesondere für grenzüberschreitend tätige natürliche Personen und Kapitalgesellschaften gleichermassen. 